\title{
Respiratory disorders of sleep
}

\begin{abstract}
This article is a short review of the different problems posed by sleep apnea syndrome. Clinical symptoms and risks to patients are recalled. It is envisaged the genetic and epidemiological aspect as well as the treatment.
\end{abstract}

Keywords: sleep respiratory disorder, sleep apnoea, sleep temporary airway obstruction, oximetry, continuous positive airway pressure
Volume 2 Issue 2 - 2018

\author{
Michel Bourin \\ Neurobiology of anxiety and mood disorders, University of \\ Nantes, France
}

Correspondence: Michel Bourin, Neurobiology of anxiety and mood disorders, University of Nantes 98, rue Joseph Blanchart, 44100 Nantes, France, Email michel.bourin@univ-nantes.fr

\section{Introduction}

The term respiratory disorder of sleep refers to a series of conditions that result in abnormal breathing during sleep. The most common is sleep apnoea.

Apnoea is a temporary break in breathing. Although there are other types of apnea, the term "sleep apnoea" is generally referred to as obstructive sleep apnoea (OSA) syndrome for which the person is unable for a short time to breathe because of temporary obstruction of the airway in the throat, called the pharynx. ${ }^{1}$ In OSAS patients, this can happen hundreds of times during a single night's sleep.

\section{Symptoms}

When we are asleep, our muscles are relaxed. In some individuals, the tongue and relaxed muscles in the throat may cause narrowing of the airways, which restricts breathing and may temporarily stop breathing. When this happens, the brain causes a brief partial awakening, which opens the airways again and resumes breathing. ${ }^{2}$ The repeated awakenings during the night can affect the quality of the sleep of important way, leading to excessive daytime sleepiness. In addition, each apnea is accompanied by a sharp increase in heart rate and blood pressure. Finally, the increase in blood pressure (hypertension) persists even when you are awake, which increases the risk of heart attack or stroke. ${ }^{3}$ Snoring usually occurs in people with OSA. The humming noise comes from tissue vibration in the throat, implying that the airways are potentially unstable and therefore more vulnerable to sagging during sleep. However, only a minority of snorers is reached by OSAS. ${ }^{4}$

OSAS symptoms can occur during the day and at night $^{5}$

I. Day
a. Excessive drowsiness
b. Lack of concentration
c. Morning headaches
d. Mood changes
e. Oral dryness
f. Impotence or loss of libido.

\section{Night}
g. Agitated sleep
h. Apnoea
i. Wake up by choking
j. Dreams of real appearance
k. Nocturia (urinate during the night)
1. Sweat
m. Insomnia
n. Salivation and grinding of teeth.

The prevalence of sleep apnea increases with age up to 60 years. Anything which makes the throat narrower than average, may increase the risk of OSAS. ${ }^{6}$

\section{Environmental factors}

Moderate or severe obesity is a major risk factor as it is accompanied by an increase in adipose tissue around the throat. Obesity is found in 60 to $90 \%$ of people with OSAS and is the most common risk factor for adults. Both tobacco and alcohol are linked to a higher prevalence of snoring and sleep apnoea. Respiratory allergies can also contribute to the appearance of OSAS especially since the patient is overweight. ${ }^{7}$

\section{Genetic factors}

Some people are more likely to develop sleep apnea because of their genes. Sleep apnea is more common in men than women. Some inherited types of bone structure of the face are associated with a narrow throat, thus increasing the risk of OSAS, the most common being having the chin fleeing. Specific genetic conditions such as Down syndrome also predispose individuals to OSAS. Recent data indicate that obstructive sleep apnoea may have a genetic basis OSAS also occurs in children and enlargement of the tonsils increases the risk of suffering from this condition. In some children or adults, severe nasal congestion may aggravate this condition. It may be considered that the syndrome will evolve if anatomical modifications of genetic origin. Such as the reduction of mandibular size and enlargement of the outer tissue tissues (tongue, lateral pharyngeal walls) .Recent evidence is accumulating about the genetic loci for these structural risk factors that predispose to the development of obstructive sleep apnoea. ${ }^{8}$ 


\section{Prevention}

Recent literature has emphasized the role of OSAS in contributing to glucose intolerance, dyslipidemia, and hypertension. If necessary, major lifestyle changes such as weight loss, reduced alcohol consumption and smoking alone can help prevent the disease. ${ }^{9}$

\section{Treatment}

The diagnosis of OSAS is often made from symptoms and clinical examination, but in most cases the overnight report is necessary to confirm this diagnosis. A detailed study of sleep, called polysomnography, records brain waves, muscle activity, eye movements, cardiac activity, chest movements, air circulation in the nose and mouth, and the oxygen level in the blood (oximetry). This detailed study is conducted in a sleep clinic, however, in most cases for which OSAS is assumed, simpler analyzes can be performed using mobile equipment, often in the patient's home. Several devices are now available to record various combinations of oxygen levels in the blood, chest movement, flow and heart rate.

The level of daytime sleepiness is assessed by a simple questionnaire called the Epworth Sleepiness Scale. He asks the patient to assess the risk of falling asleep in different everyday situations. Symptoms of sleep apnea, such as snoring and observed apnea, may also be reported by the person sharing the same bed.

The most effective treatment for sleep apnoea is Continuous Positive Airway Pressure (CPAP), which is simple and highly costeffective. This form of treatment includes an apparatus that generates a flow of air through a mask on the nose (or nose and mouth) at a pressure that is adjusted to keep the throat open during the night. ${ }^{10}$ For a persistent benefit, this device should be used every night. There are other options, including a device worn inside the mouth to bring the lower jaw forward or, if necessary, surgery to remove the tonsils. There is currently no pharmacological treatment

\section{Epidemiology}

In developed countries sleep apnea is reported to be between 3\% and $7 \%$ of middle-aged men and $2-5 \%$ of women. Sleep apnea is associated with an increased risk of hypertension, as well as heart disease, depression and drowsiness. People with untreated sleep apnea have a 1.2 to 2 time higher risk of a car accident. The pre-diagnosis of sleep apnoea is associated with costs that increase per capita health care by $50 \%$ to $100 \%$. Prevalence of OSAS increases with age. ${ }^{11}$

\section{Current and future}

Better awareness is needed regarding the disease and its consequences if not treated. National transport and health authorities need to recognize the effects of OSAS drowsiness on driving to reduce risks for those concerned and the wider public More effort is needed to simplify tests to diagnose disease Infrastructure for sleep apnoea treatment needs to be expanded as wait times for assessment and treatment in Europe are a major problem. ${ }^{12}$ There is a need to better understand the treatments that work best on different groups of people, to improve their effectiveness.

\section{Acknowledgements}

None.

\section{Conflicts of interest}

The authors declared that there are no conflicts of interest.

\section{References}

1. Jordan AS, McSharry DG, Malhotra A. Adult obstructive sleep apnoea Lancet. 2014;383(9918):736-747.

2. Jun JC, Chopra S, Schwartz AR. Sleep apnoea. Eur Respir Rev. 2016;25(139):12-18.

3. Melamed KH, Goldhaber SZ. Cardiology Patient Page. Obstructive Sleep Apnea. Circulation. 2015;132(6):e114-e116.

4. Ramar K, Dort LC, Katz SG, et al. Clinical Practice Guideline for the Treatment of Obstructive Sleep Apnea and Snoring with Oral Appliance Therapy: An Update for 2015. J Clin Sleep Med. 2015;11(7):773-827.

5. Contencin P, Guilleminault C, Manach Y. Long-term follow-up and mechanisms of obstructive sleep apnea (OSA) and related syndromes through infancy and childhood. Int $J$ Pediatr Otorhinolaryngol. 2003;67(Suppl 1):S119-S123.

6. Maspero C, Giannini L, Galbiati G, et al. Obstructive sleep apnea syndrome: a literature review. Minerva Stomatol. 2015;64(2):97-109.

7. Kent DT, Soose RJ. Environmental factors that can affect sleep and breathing: allergies. Clin Chest Med. 2014;35(3):589-601.

8. Schwab RJ. Genetic determinants of upper airway structures that predispose to obstructive sleep apnea. Respir Physiol Neurobiol. 2005;147(2-3):289-298.

9. Montesi SB, Bajwa EK, Malhotra A. Biomarkers of sleep apnea. Chest. 2012;142(1):239-245

10. Nicolini A, Banfi P, Grecchi B, et al. Non-invasive ventilation in the treatment of sleep-related breathing disorders: A review and update. Rev Port Pneumol. 2014;20(6):324-335.

11. Trzepizur W, Gagnadoux F. Epidemiology of obstructive sleep apnoea syndrome. Rev Mal Respir. 2014;131:568-577.

12. Lévy P, Tamisier R, Minville $C$, et al. Sleep apnoea syndrome in 2011: current concepts and future directions. Eur Respir Rev. 2011;20(121):134-146. 